Ann. Sci. forest., 1969, 26 (4), 519-524.

NOTE TECHNIQUE

\title{
ABSCISSION DES AIGUILLES D'ABIES ALBA (MILLER) SOUS L'ACTION DE L'ÉTHYLÈNE. APPLICATION POUR LA DÉTERMINATION DE LA MASSE FOLIAIRE
}

\author{
A. RIEDACKER \\ Station de Sylviculture et de Production, \\ Centre national de Recherches forestières, 54-Nancy \\ Institut national de la Recherche agronomique.
}

\section{SOMMAIRE}

L'auteur met en évidence l'action de l'éthylène sur l'abscission des aiguilles d'Abies alba (=A. pectinata D.C.); l'abscission des aiguilles âgées se fait plus rapidement que celle des jeunes aiguilles. Le traitement n'affecte pas leurs poids.

La méthode peut être utilisée pour la détermination des masses foliaires.

\section{INTRODUCTION}

Pouvoir évaluer la masse et la surface foliaire des arbres revêt une importance extrême tant en physiologie qu'en écologie forestière; connaissant ces valeurs, on peut en effet établir des bilans de photo-synthèse, les cycles biogéochimiques des éléments minéraux et de nombreux indices, tels que l'indice foliaire (rapport de la surface foliaire à la projection de celle-ci sur le sol), le rapport masse de feuilles/masse ligneuse, etc...

La détermination de la masse sèche des aiguilles d'Epicea ne présente pas de difficultés majeures; les aiguilles se désarticulent de la branche après dessiccation par simple choc. Chez Abies alba, les vaisseaux et éléments de raccords entre le rameau ligneux et l'aiguille sont plus résistants et après dessiccation un simple choc mécanique ne suffit pas pour séparer les aiguilles du rameau.

Défolier manuellement des branches de sapin serait un travail gigantesque, puisqu'on estime que pour un beau houppier, il faudrait environ 200 jours de travail! 
Nous avons donc envisagé diverses méthodes visant à provoquer l'abscission des aiguilles, c'est-à-dire l'apparition à la base du pétiole d'une zone génératrice de type subérophellodermique qui produit du liège du côté rameau et du côté de l'aiguille des cellules fragiles dont la membrane se gélifie, et qui constituent la couche séparatrice. Lorsque cette couche est formée, l'aiguille n'est alors plus retenue que par les faisceaux conducteurs, et un petit choc provoque sa chute.

Parmi les différentes méthodes permettant de déclancher l'abscission, nous avons retenu celle utilisant l'action de l'éthylène qui nous a paru être la plus élégante.

Les deux points suivants sont étudiés dans la présente note:

- d'une part, l'efficacité de l'éthylène sur l'abscission des aiguilles des branches de sapins coupées ;

- d'autre part, les variations de poids consécutives au traitement.

\section{MATERIEL ET METHODE}

La figure 1 montre que le poids sec de 100 aiguilles de différentes pousses varie considérablement :

— en fonction de l'âge; lorsqu'elles vieillissent leur poids augmente. Ceci est particulièrement net pour les aiguilles de la base du houppier ;

- en fonction du niveau de prélèvement des échantillons dans la couronne; les aiguilles d'ombre à la base du houppier étant les plus légères ;

- dans certains cas, l'orientation de la branche par rapport aux points cardinaux doit également être prise en considération ; c'est pourquoi nous avons indiqué pour chaque branche le $\mathrm{n}^{\circ}$ du verticille compté à partir de la base du houppier et son orientation.

Des considérations précédentes découle la façon dont nous avons prélevé les échantillons; le 15 novembre 1968, nous avons récolté des pousses A et B, de même âge, situées symétriquement sur une même branche, ceci afin d'avoir des poids secs de 100 aiguilles voisins (cf. fig. 2). Parmi les 35 couples d'échantillons, 17 étaient de l'année 1968, 11 de l'année 1967 et 7 des années 1966 et 1965.

Les pousses B ont été disposées dans des sachets de polyéthylène remplis d'un gaz commercial ( $5 \%$ d'éthylène et le complément en azote) et d'un peu d'air (oxygène). La température du local d'expérience oscillait entre 10 et $15^{\circ} \mathrm{C}$.

Les aiguilles des pousses $\mathrm{A}$ ont été séchées à l'étuve immédiatement après la récolte, à $70^{\circ} \mathrm{C}$ durant 24 heures. Les aiguilles des pousses $\mathrm{B}$ ont subi le même traitement après abscission. Notons qu'aucune abscission n’a eu lieu sur des échantillons témoins.

\section{RESULTATS}

1) Labscission des aiguilles se fait plus ou moins rapidement en fonction de lâge.

Les aiguilles âgées tombent beaucoup plus rapidement que les jeunes aiguilles (fig. 3) : la différence étant surtout nette entre les aiguilles âgées d'un an et les aiguilles plus vieilles.

GaWady et Avery (1950) ont proposé un mécanisme d'abscission à base d'auxine et d'éthylène. Hall et Morgan (1964) ont de leur côté suggéré que l'éthylène pourrait stimuler l'action des auxines-oxydases !

Or, selon PILet (1961), la teneur en auxine-oxydases (du moins dans les organes tels que les racines de Lens) augmenterait au fur et à mesure que les tissus vieillissent. Ceci est peut-être une explication à la chute plus rapide des vieilles aiguilles par rapport aux aiguilles de l'année. 


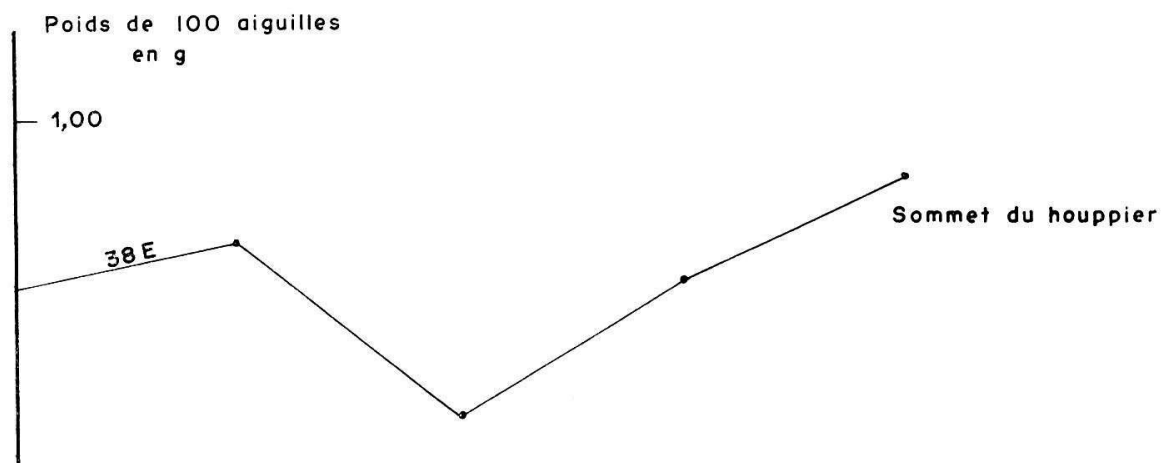

0,80

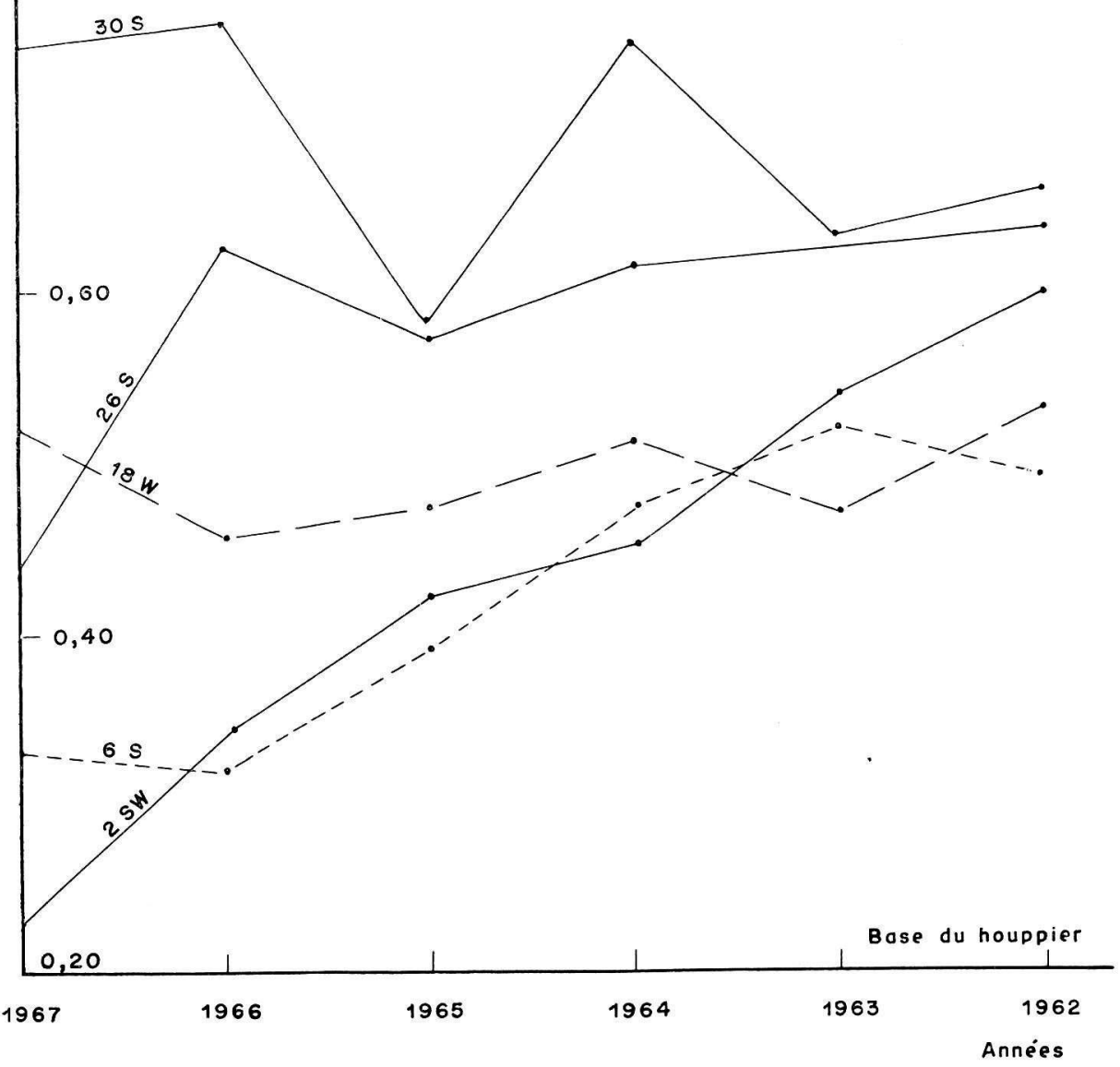

FIG. 1. - Variations du poids sec de 100 aiguilles en fonction de leur âge et de leur position dans la couronne; les chiffres indiquent le numéro du verticille compté à partir de la base du houppier et les lettres l'orientation do la branche par rapport aux noints cardinciux. 
2) La comparaison des échantillons non traités et de ceux ayant subi l'action de l'éthylène n'a révélé aucune différence significative de poids.

Abeles et Coll (1967) ont aussi montré que chez Phaseolus vulgaris, l'éthylène stimule l'abscission sans entraîner aucun des phénomènes de dégradation associés à la sénescence.

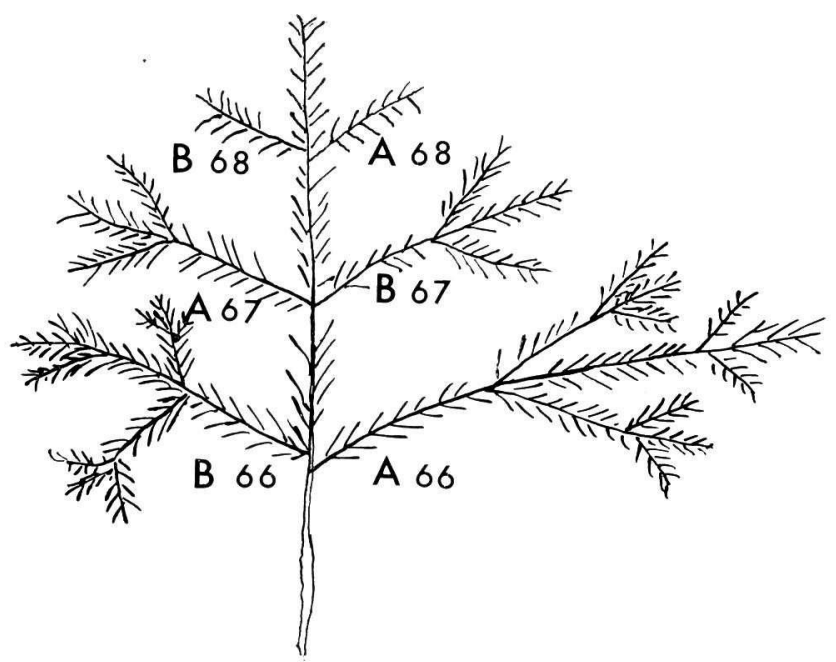

FIG. 2. - Mode de prélèvement des couples d'échantillons de pousses $A$ et B

\section{APPLICATION PRATIQUE}

Selon qu'on cherchera à évaluer avec précision la masse foliaire d'un arbre, ou les masses foliaires de plusieurs arbres, on procédera différemment.

Dans le premier cas, encore que l'opération soit fastidieuse, on peut penser à une mesure directe de la masse foliaire, ou bien faire un échantillonnage des branches de l'arbre.

Dans le second cas, on cherchera à établir des équations de régression entre des paramètres facilement mesurables tels que le diamètre de la branche, la hauteur de la branche dans l'arbre et la masse foliaire des branches.

Quelle que soit la méthode retenue, on devra mesurer directement la masse foliaire d'un certain nombre de branches, donc séparer les aiguilles des rameaux ligneux. Dans le cas d'Abies alba, nous suggérons de procéder de la façon suivante:

après abattage de l'arbre, on coupe à l'aide d’un sécateur toutes les parties feuillées d'une branche, on les dispose dans un sac en polyéthylène (épaisseur 5/100) que l'on remplit ensuite avec du gaz commercial (azote + éthylène). Le nombre de sacs nécessaire par branche est bien sûr fonction des dimensions du sac et de la branche.

Quelques précautions sont cependant nécessaires:

- il est préférable de remplir des sacs au plus à la moitié de leur capacité totale pour éviter des risques de moisissures;

- il est aussi indispensable de laisser pénétrer un peu d'air afin de ne pas priver les branchages d'oxygène. 


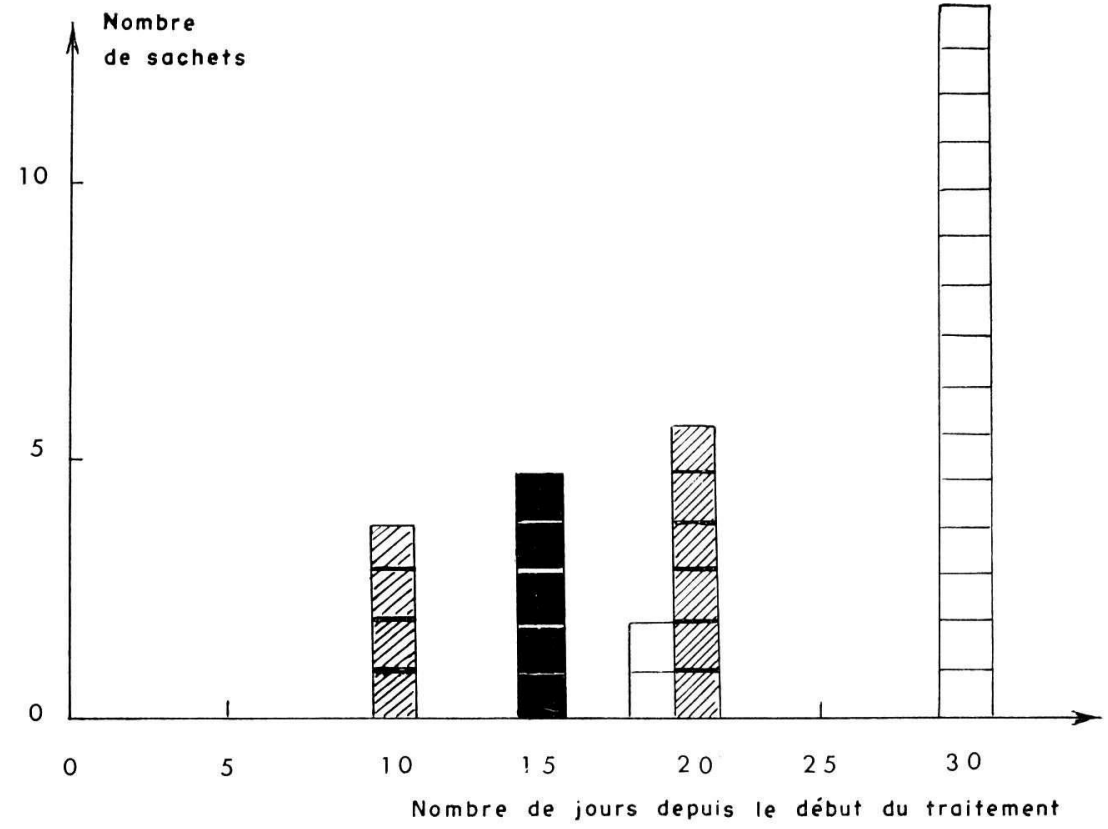

Sachets d'aiguilles de 1968

1967

1966 et 1965

FIG. 3. - Durée du traitement nécessaire pour provoquer l'abscission des aiguilles

A ce sujet, Abeles (1968) vient de montrer que lorsque la teneur en oxygène est inférieure à $20 \%$, l'abscission se fait plus lentement et même pas du tout en l'absence d'oxygène. Un excédent d'oxygène en revanche ne stimule pas l'abscission.

Reçu pour publication en octobre 1969.

\author{
SUMMARY \\ ABSCISSION OF Abies alba NEEDLES WITH ETHYLEN. \\ UTILIZATION TO ASSESS THE FOLIAGE WEIGHT OF FIR TREES.
}

The action of ethylen on the abscission of fir needles of cut branches has been investigated with success. Older needles need less time to get separated from the branches than younger needles. The treatment did not affect the weight of the needles. Consequently the method can be used to assess the mass of fir needles. 


\section{ZUSAMMENFASSUNG}

ABSZISSION DER Abies alba NADELN DURCh ATHYLEN.

ANWENDUNG Für DIE ABSChäTZUNG DES NADELgEWichts DER TANNE.

Der Einfluss von Athylen auf die Abszission der Tannennaldeln ist mit Erfolgs versucht worden. Jüngere Nadeln branchen mehr Zeit um alzufallen als ältere Nadeln. Das Verfahren verändert das Gewicht der Nadeln nicht. Deswegen kann mann diese Methode für Abschätzung des Nadelgewichts der Tanne anwenden.

\section{REFERENCES BIBLIOGRAPHIQUES}

Addicot F. T., Lynch R. S., 1955. Physiology of abscission. Annu. Rev. Plant Physiol., 6, 211-238.

Abeles F. B., Holm, Cahagan, 1967. Abscission; the role of senescence. Sixth Int. Conf. on Plant Growth Subst., Carleton University, Book of Abstracts.

Abeles F. B., Cahagan, 1968. Abscission; the ro'e of ethylene and ethylen z analogues, carbon dioxide and oxygen. Plant Physiol., 43, 1255-1258.

Carns, 1966. Abscission and its control. Annu. Rev. Plant Physiol., 17, 295-314.

Gawady, Avery, 1950. Amer. J. Bot., 37, 172-180.

Hall, Morgan, 1964. Régulateurs naturels de la croissance végétale, Paris, C.N.R.S., 727-45.

Pilet P. E., 1961. Les phytohormones de croissance. Méthodes, Chimie, Biochimie, Physiologie, Paris, Masson, 350-352, 572-574. 